\title{
Wissenschaftliche Politikberatung: Chancen der Digitalisierung nutzen
}

Im April 2019 habe ich die Aufgabe des Chefredakteurs für die Zeitschrift Wirtschaftsdienst übernommen. Dabei kann ich auf Leistungen meiner Vorgängerin Brigitte Preissl und der Redaktion sowie der Autorinnen und Autoren aufbauen, die den Wirtschaftsdienst zu dem gemacht haben, was er ist: eine traditionsreiche und unabhängige Säule der deutschsprachigen wirtschaftspolitischen Debatte und eine Brücke zwischen wissenschaftlicher Forschung und politischer Praxis. Der Wirtschaftsdienst wurde im Jahr 1916 gegründet und gehört heute zu den wenigen wirtschaftswissenschaftlichen Fachzeitschriften, die in deutscher Sprache erscheinen. Die deutsche Sprache ermöglicht dabei eine intensive Diskussion der institutionellen Rahmenbedingungen der deutschen Wirtschaftspolitik. Zudem soll der wissenschaftliche Diskurs zu aktuellen und relevanten Themen der Politikberatung gefördert und dabei verschiedenen theoretischen Denkschulen eine Plattform geboten werden. Viele namhafte Autoren haben im Wirtschaftsdienst veröffentlicht, darunter 14 Wirtschaftsminister der Nachkriegszeit und zwei spätere Bundeskanzler, sowie nicht zuletzt der britische Ökonom John Maynard Keynes mit Aufsätzen unter anderem zu den Reparationszahlungen nach dem ersten Weltkrieg, dem Goldstandard und der Weltwirtschaftskrise. Seit jeher widmet sich der Wirtschaftsdienst den aktuellen politischen Debatten und Herausforderungen.

Eine der wesentlichen Herausforderungen unserer Zeit ist die Digitalisierung. Sie ist mit weitreichenden ökonomischen Konsequenzen verbunden, aber auch mit Konsequenzen für die Wirtschaftswissenschaft. Ähnlich wie frühere technische Revolutionen macht es auch die Digitalisierung erforderlich, eine Infrastruktur bereitzustellen, die nicht notwendigerweise vom Markt in gesellschaftlich optimaler Weise erzeugt werden kann. Durch die Digitalisierung lassen sich Informationsasymmetrien deutlich leichter beheben als vor wenigen Jahren. Man könnte daher meinen, dass wir der Annahme vollständiger Informationen der neoklassischen Ökonomik ein Stück weit näher sind als zuvor. Die Kosten für Informationen und Kommunikation sinken mit einer Geschwindigkeit, dass der hieraus gewonnene gesellschaftliche Nutzen nur erahnt werden kann. Denn wie soll der Konsum von nun weitgehend kostenlosen Informationen, die zum Allgemeingut geworden sind, bewertet werden? Wie viel mehr Informationen werden heute im Vergleich zu den 1990er Jahren konsumiert, investiert oder exportiert? Wie gehen Informationen als freie Güter in das Bruttoinlandsprodukt oder die nationale Wohlfahrtsmessung ein? Die Wohlfahrtsforschung hat seit geraumer Zeit darauf hingewiesen, dass die Orientierung am Bruttoinlandsprodukt als Wohlstandsmaß viele Bereiche des gesellschaftlichen Lebens ignoriert. Ebenso kann die Wohlfahrtswirkung bzw. Produktivität der zu Grenzkosten von Null produzierten und teilweise kostenfrei zugänglichen Produkte allenfalls geschätzt werden. Für zunehmend frei zugängliche Informationen verlieren Preise als Qualitätssignal ihre Wirkung. Eine marktbasierte Bewertung scheidet somit aus. Die Logik der Digitalisierung scheint zumindest Teile der auf Knappheit gebauten Wirtschaftstheorie außer Kraft zu setzen.

Die Digitalisierung bietet Anknüpfungspunkte für Diskussionen zur Wirtschaftspolitik. Sie führt zu einem Strukturwandel, der Gewinner und Verlierer mit sich bringt und es notwendig macht, die Gewinne der Digitalisierung angemessen zu verteilen bzw. die Verlierer des Strukturwandels entsprechend zu kompensieren. Grenzkosten von Null führen zu natürlicher Monopolbildung. Für die Wirtschaftspolitik stellt sich die Frage, wie mit der Bildung natürlicher Monopole auf digitale Produkte,

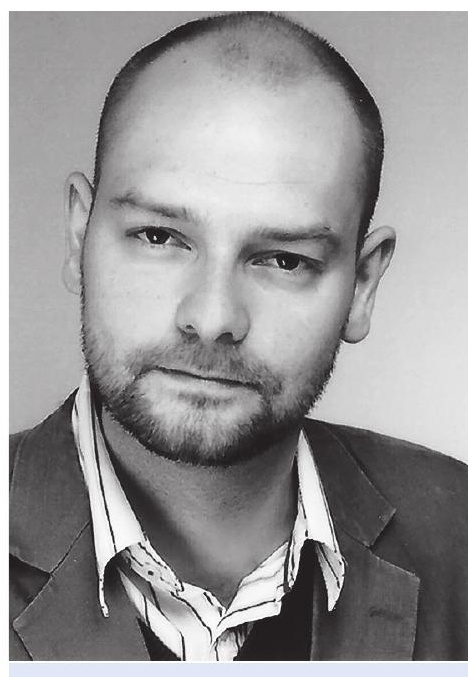

Christian Breuer ist

Chefredakteur von

Wirtschaftsdienst

und Intereconomics

in der ZBW - Leibniz-

Informationszentrum Wirt-

schaft - in Hamburg. 
Daten und Netzwerke umgegangen werden soll. Dabei erschweren internationale Monopole den nationalen Akteuren die Regulierung, sodass es schwieriger werden dürfte, funktionsfähige Marktprozesse zu gewährleisten oder von vornherein ein effizientes Marktergebnis vorauszusetzen. Die Bedrohung der heimischen Wirtschaft durch internationale Monopole oder Champions in den USA oder China hat auch Wirtschaftsminister Peter Altmaier veranlasst, eine Debatte um eine neue Industriepolitik und die Förderung nationaler oder europäischer Industrien anzustoßen.

Für die Regional- und Strukturpolitik könnte die Digitalisierung eine Chance bedeuten. Waren die ländlichen Regionen früher räumlich von den Zentren abgeschnitten, können diese nun völlig transaktionskostenfrei mit den Zentren kommunizieren. Die Kosten der Anbindung ländlicher Regionen reduzieren sich darauf, die Infrastruktur für den ländlichen Raum bereitzustellen. Gerade für die ländlich geprägten und vom Strukturwandel stark beeinträchtigten Umlandregionen von Metropolen wie Berlin könnte der schnelle Ausbau der Breitbandinfrastruktur mit erheblichen positiven Externalitäten verbunden sein. Aktuell leiden die Zentren unter überspannten Immobilienmärkten; eine geeignete Infrastrukturpolitik könnte helfen, den ländlichen Raum stärker anzubinden, und Teile der Verspannungen in den asymmetrischen Mietpreisbewegungen zwischen Zentrum und Umland könnten gelöst werden. Wird diese Chance verpasst, verstärken sich allerdings die Hypotheken für den ländlichen Raum, regionale Konzentrationsprozesse nehmen zu, mit entsprechenden Folgen.

Viele dieser Themen haben den Wirtschaftsdienst in der Vergangenheit beschäftigt. Es gab Zeitgespräche und Aufsätze zur Industriepolitik und zu verschiedenen Aspekten der Digitalisierung sowie Konferenzen unter anderem zur Regionalpolitik. 2019 werden wir eine Konferenz zu den digitalen Potenzialen anbieten. Auch für die Wirtschaftswissenschaft als Disziplin hat die Digitalisierung Konsequenzen. So gewinnen Bereiche der Ökonomik, deren Gegenstand die Nutzung statistischer Verfahren zur elektronischen Datenverarbeitung ist, wie die empirische Wirtschaftsforschung bzw. Ökonometrie, an Bedeutung. Wirtschaftspolitische Empfehlungen und Erkenntnisse werden zunehmend auf Basis dieser empirischen Wirtschaftsforschung gegeben, was unter dem Schlagwort „evidenzbasierte Politikberatung“ zusammengefasst wird. Dabei ist von zentraler Bedeutung, dass Daten und Erkenntnisse transparent aufbereitet werden, damit die Ergebnisse insgesamt replizierbar gemacht werden. Ohne diese Nachvollziehbarkeit durch Open-Data- bzw. OpenScience-Politik bliebe der wissenschaftliche Diskurs zur Politikberatung intransparent und eine Black Box für die politischen Akteure.

Auch dem Wirtschaftsdienst bietet Open Science viele Möglichkeiten. So lässt sich die Reichweite der Beiträge digital erweitern: Die Beiträge der Autorinnen und Autoren können durch die Präsenz im Netz, auf der Website, über den Twitter-Account und über die Zusammenarbeit mit ökonomischen Blogs (Herdentrieb, Ökonomenstimme etc.) einem größeren Leserkreis vermittelt werden. Dies wird in Zukunft noch umfassender möglich sein: Ab dem Jahr 2020 wird der Wirtschaftsdienst Open Access, also digital frei zugänglich sein, damit alle Leser sofort kostenfrei auf alle Artikel zugreifen können. Da die Adressaten für wissenschaftliche Informationen zur Wirtschaftspolitik nicht nur Wissenschaftler sind, sondern auch Journalisten, Politiker, Entscheidungsträger und interessierte Bürger, sollte der Zugang für wissenschaftliche Auseinandersetzungen zur Wirtschaftspolitik nicht auf

Christian Breuer ZBW - Leibniz-Informationszentrum Wirtschaft c.breuer@zbw.eu wissenschaftliche Einrichtungen beschränkt bleiben. Der Wirtschaftsdienst wird daher - auch und gerade in Zeiten verbreiteter „Fake News“ - Wissenschaftlern eine Plattform bieten, ihre Forschung offen in Politik, Medien und Gesellschaft zu kommunizieren. 